UAB-FT-598

FTUAM-06-02

IFT-UAM/CSIC-06-10

DESY-08-167

MPP-2008-158

GEF-TH-4-08

\title{
Mixing of photons with massive spin-two particles in a magnetic field
}

\author{
Carla Biggio ${ }^{1,2,3}$, Eduard Massó ${ }^{4}$ and Javier Redondo ${ }^{4,5}$ \\ ${ }^{1}$ Departamento de Física Teórica, Universidad Autónoma de Madrid, Spain \\ ${ }^{2}$ Max-Planck-Institut für Physik, München, Germany \\ ${ }^{3}$ Dipartimento di Fisica, Università di Genova, Italy \\ ${ }^{4}$ Institut de Física d'Altes Energies, Universitat Autònoma de Barcelona, Spain \\ ${ }^{5}$ Deutsches Elektronen-Synchrotron DESY, Hamburg, Germany
}

\begin{abstract}
We study the mixing of photons with hypothetical massive spin-two particles in the presence of a magnetic field. Mixing phenomena have been studied in the case of axion-like particles and strictly massless spin-two particles (gravitons) but not in this case. We find several interesting differences between them.
\end{abstract}

\section{Introduction}

A photon traveling through an electromagnetic field may convert into neutral particles that have a coupling to two photons. The process has been observed in the laboratory; indeed, photons in the electric field of a nucleus produce pions as predicted by Primakoff [1]. If the particle is light enough we expect that the mixing of the photon with the particle leads to a coherent superposition of the two. Such an effect is at the heart of the proposals to look for axions, since coherence enhances the probability of axion-photon conversion [2]. All current axion searches are based on detection 
techniques that exploit the increase in the sensitivity that this mixing phenomenon provides. These searches include haloscopes [3], helioscopes [4, 5, 6, 7, 8, 9] and laser experiments [10, 11, 12, 13, 14, 15, 16, 17, 18, 19, 20] (for a review see Ref. [21]).

Since the axion is a theoretically well motivated particle, almost all mixing studies have focused on this case. Of course any other light spinless particle coupling to photons could lead to the same phenomenology [22]. Furthermore, photons may oscillate also into particles with spin higher than one (mixing with spin-one particles is forbidden due to the Landau-Yang theorem [23, 24]), the most prominent case being the graviton.

Photon-graviton mixing has being considered in the framework of standard fourdimensional general relativity [25, 26, 27, 28, 29, 30], where gravitons are massless, and also in the context of extra-dimensions [31] where, in addition to the massless mode, one finds a Kaluza-Klein tower of massive gravitons. Unfortunately, due to the extreme smallness of Newton's constant, the effects of photon-graviton mixing are usually of little relevance.

In this paper we want to present our calculations for photon mixing with a generic massive spin-two particle, in principle unrelated to Kaluza-Klein gravitons.

In our study we have seen that there are some peculiarities in the spin-two case that are not present when the mixing is with pseudoscalars such as axions; we consider instructive to present such differences. Even more than that, we have realized that even if the mass $m$ of the spin-two particle is small, there are significant differences with the strictly massless case. Specifically, we find that there is no decoupling, in the sense that in the $m \rightarrow 0$ limit there are effects not present in the massless theory. The helicity-zero component of the massive theory contributes without suppression to the observables we calculate.

Our results may be useful for a certain number of experiments on laser propagation in magnetic fields [10, 11, 12, 13, 14, 15, 16, 17, 18, 19, 20]. Indeed, photon mixing leads to several effects and it is of interest whether we may interpret or not such potential effects as the result of the mixing of photons with spin-two particles. Again, the finding related to the contribution of the helicity-zero component makes this interpretation as natural as the spinless one, in the potential case that effects are found experimentally. However, one should always bare in mind that these couplings are subject to strong constraints not only from astrophysics [4, 5, 6, 7, 8, 9, 32, 33, 34], which is actually the case of axions, but also from the usually very demanding laboratory searches for long range forces [35, 36, 37]. Some refined models that evade constraints from astrophysics and/or searches for new long range forces have been developed [38, 39, 40, 41], but they all suffer from some lack of naturalness.

Last but not least our work might be of interest concerning the controversy of the massless limit of a theory of massive gravity (see [42] for a recent review). The issue goes back to the study of van Dam and Veltman [43] and Zakharov [44, who showed 
that there was a discontinuity in the limit of the graviton mass going to zero when compared with the usual gravitational theory with a massless graviton, and to the study of Vainshtein [45], who argued that the non-linearity of Einstein equations could solve the problem. There has been much work on such topic and, as far as we know, there is no clear consensus. It is not our intention to enter into the intricacies of such deep problems, but rather to present a calculation that shares with gravitation the fact that we also deal with a spin-two particle. In our case, which by the way is developed in linear theory, we find a discontinuity in the $m \rightarrow 0$ limit.

In this paper we shall not review the pseudoscalar case; the work of Raffelt and Stodolsky [30] is very complete and can be complemented with more recent studies [46, 47]. Instead we will present in Section 2 the mixing of photons with scalar particles. This will help us in fixing some of the notations and conventions we use. Also, another motivation is that there are small technical differences between the scalar and the pseudoscalar cases, unnoticed in previous literature [48, 49], which will also appear in the spin-two calculation. Section 3 is devoted to the spin-two calculation and a final discussion is presented in Section 4. Some technical details on how we choose the polarization basis and the demonstration of an useful identity are shown in the appendixes.

\section{Mixing of Photons with Scalars}

The Lagrangian describing a scalar field $\phi$ coupled to two photons with coupling constant $g_{0}$ is

$$
\mathcal{L}=\mathcal{L}_{E M}+\mathcal{L}_{K G}+\frac{1}{4} g_{0} F^{\mu \nu} F_{\mu \nu} \phi
$$

where $F_{\mu \nu}$ is the electromagnetic field strength and we have the usual free parts

$$
\begin{aligned}
\mathcal{L}_{E M} & =-\frac{1}{4} F^{\mu \nu} F_{\mu \nu} \\
\mathcal{L}_{K G} & =\frac{1}{2}\left(\partial_{\alpha} \phi\right)\left(\partial^{\alpha} \phi\right)-\frac{1}{2} m_{0}^{2} \phi^{2} .
\end{aligned}
$$

We use the metric $\eta_{\mu \nu}=\operatorname{Diag}\{1,-1,-1,-1\}$. Following the mixing formalism of Ref. [30], we decompose $F_{\mu \nu}$ as

$$
F_{\mu \nu}=F_{\mu \nu}^{e x t}+\partial_{\mu} A_{\nu}-\partial_{\nu} A_{\mu}
$$

where $F_{\mu \nu}^{e x t}$ represents the external field and $A_{\mu}$ the propagating quantum photon field. The mixing equations in the Lorentz gauge and considering the external magnetic field constant are:

$$
\begin{aligned}
\left(\partial^{2}+m_{0}^{2}\right) \phi & =\frac{1}{2} g_{0} F_{\mu \nu}^{e x t}\left(\partial^{\mu} A^{\nu}-\partial^{\nu} A^{\mu}\right) \\
\partial^{2} A_{\nu} & =g_{0} F_{\mu \nu}^{e x t} \partial^{\mu} \phi .
\end{aligned}
$$


We choose the $z$-axis as the direction of propagation of light and the magnetic field as $\vec{B}=\left(B_{T}, 0, B_{L}\right)=\left(F_{23}^{e x t}, 0, F_{12}^{e x t}\right)$. We also have $A_{\mu}=(0 ; \vec{A})=\left(0 ; A_{\|}, A_{\perp}, 0\right)$, with polarizations $A_{\|}$and $A_{\perp}$ respectively parallel and perpendicular to $B_{T}$.

We will work in the case in which $m_{0}$ is small and the magnetic field is stationary and slowly varying in space, which are the conditions typically interesting for laboratory experiments. The motivation of the first requirement is two-fold. On the one hand $m_{0}$ has to be smaller than the photon energy because otherwise real $\gamma-\phi$ transitions can not occur. On the other hand if $m_{0}^{2} / 2 \omega^{2} \ll 1$ ( $\omega$ is the energy) we can linearize the equations of motion, which simplifies the calculation without loosing relevant information. Moreover, we consider $g_{0}$ small enough so that we can work at first non-trivial order 1 .

With all these conditions satisfied, the field solutions to the equations of motion in the stationary regime will be plane waves with an energy dependence $e^{i \omega t}$ and a spatial dependence $e^{-i p z}$ to be determined 2. In the relativistic case in which $m_{0} \ll \omega$ we expect $|p| \simeq \omega$ and we can linearize the operator $\partial^{2}$ in the equations of motion:

$$
\omega^{2}+\partial_{z}^{2}=\left(\omega+i \partial_{z}\right)\left(\omega-i \partial_{z}\right)=(\omega+p)\left(\omega-i \partial_{z}\right) \simeq 2 \omega\left(\omega-i \partial_{z}\right) .
$$

By reducing the order of the system of differential equations to one we are loosing the possibility of imposing boundary conditions on the field derivatives and thus of considering reflected waves. In turn this can be used to argue that the amplitudes of such waves should be of the order of the neglected terms when we linearize as in Eq. (7), i.e. suppressed by a factor $(\omega-p) / \omega$ at least. Explicit formulas for the case of the axion can be found in Ref. [46, 47].

We therefore obtain the following equations:

$$
\begin{aligned}
\left(\omega-i \partial_{z}-\frac{m_{0}^{2}}{2 \omega}\right) \phi+\frac{g_{0} B_{T}}{2 \omega} \partial_{z} A_{\perp} & =0 \\
\left(\omega-i \partial_{z}+\Delta_{\perp}\right) A_{\perp}-\frac{g_{0} B_{T}}{2 \omega} \partial_{z} \phi & =0 \\
\left(\omega-i \partial_{z}+\Delta_{\|}\right) A_{\|} & =0 .
\end{aligned}
$$

We have introduced the parameters $\Delta_{\perp}$ and $\Delta_{\|}$to take into account that photons may travel in a medium with an index of refraction $n_{i} \simeq 1+\Delta_{i} / \omega$ and again we are assuming $\Delta_{i} \ll \omega$.

\footnotetext{
${ }^{1}$ From an effective field theory point of view, we can consider the $\phi \gamma \gamma$ coupling in Eq. (11) as being generated by new physics related to an energy scale $\propto g_{0}^{-1}$. Therefore, going beyond the lowest nontrivial order in $g_{0}$ would be probably inconsistent since, whatever the new physics is, it will most likely generate other effective interactions with couplings proportional to higher powers of $g_{0}$ which would have comparable impact on the final results.

${ }^{2}$ Given the way we chose the axes, $x$ and $y$ play no role.
} 
We notice that only magnetic fields transverse to the direction of propagation of light lead to mixing effects in this case. This can be understood since when the external magnetic field is longitudinal we have azimuthal symmetry along the propagation axis so the $z$-component of the angular momentum must be conserved. Since the photon is helicity-one it cannot convert into a scalar particle.

Let us now turn our attention to the resolution of the equations of motion. The polarization $A_{\|}$is solved trivially (from now on we drop the temporal dependence $e^{i \omega t}$, common to all fields):

$$
A_{\|}(z)=e^{-i \omega n_{\|} z} A_{\|}(0) .
$$

Let us write the equations of the $A_{\perp}-\phi$ system in matricial form

$$
\left[\omega-i \partial_{z}+\left(\begin{array}{cc}
0 & a \\
a & 0
\end{array}\right)\left(i \partial_{z}\right)+\left(\begin{array}{cc}
-\Delta_{0} & 0 \\
0 & \Delta_{\perp}
\end{array}\right)\right]\left(\begin{array}{c}
\phi \\
A_{\perp}
\end{array}\right)=0
$$

where, for convenience, we have performed the shift $A_{\perp} \rightarrow i A_{\perp}$ and we have defined

$$
a=\frac{g_{0} B_{T}}{2 \omega} \quad, \quad \Delta_{0}=\frac{m_{0}^{2}}{2 \omega} .
$$

To solve for the $A_{\perp}-\phi$ mixing, we search for solutions $A_{\perp}(z)=\widetilde{A}_{\perp} e^{-i p z}$ and $\phi(z)=$ $\widetilde{\phi} e^{-i p z}$ and are led to the following matricial equation in Fourier space,

$$
\left(\begin{array}{cc}
\omega-p-\Delta_{0} & a p \\
a p & \omega-p+\Delta_{\perp}
\end{array}\right)\left(\begin{array}{c}
\widetilde{\phi} \\
\widetilde{A}_{\perp}
\end{array}\right)=\left(\begin{array}{l}
0 \\
0
\end{array}\right) .
$$

In order to have non-trivial solutions, the determinant of the matrix above should vanish. This requirement implies an equation for $p$, with two solutions: $p_{1}$ and $p_{2}$. Introducing the values $p=p_{1}, p_{2}$ in Eq. (14) we find that the eigenvectors $\left(\widetilde{\phi}, \widetilde{A}_{\perp}\right)$ corresponding to these solutions satisfy

$$
\frac{\widetilde{A}_{\perp}^{(1)}}{\widetilde{\phi}^{(1)}}=\frac{-a p_{1}}{\omega-p_{1}+\Delta_{\perp}} \equiv-\tan \Theta_{1} ; \frac{\widetilde{\phi}^{(2)}}{\widetilde{A}_{\perp}^{(2)}}=\frac{-a p_{2}}{\omega-p_{2}-\Delta_{0}} \equiv \tan \Theta_{2} .
$$

The definition of the angles $\Theta_{1}$ and $\Theta_{2}$ is such that they measure the angular distance (in this flavor space) of the solution 1 to the pure $\phi$ state $(1,0)$ and of the solution 2 to the pure photon $(0,1)$ state.

At this level we already find a formal difference with the pseudoscalar case studied in Ref. [30]. The off-diagonal matrix elements of Eq. (14) involve the wavenumber $p$ while in the pseudoscalar case involve the frequency $\omega$; therefore in general $\Theta_{1} \neq \Theta_{2}$, unlike in the pseudoscalar case 3 . However, one can show that

$$
\frac{\tan \Theta_{1}}{\tan \Theta_{2}}=\frac{\omega-\Delta_{0}}{\omega+\Delta_{\perp}}
$$

\footnotetext{
${ }^{3}$ Let us stress that this conclusion still holds even if we do not linearize the equations of motion.
} 
and therefore, in the relativistic approximation considered here 4 , we can safely take both angles to be equal.

We will content ourselves showing here the explicit formulas in the limits

$$
\Delta_{0},\left|\Delta_{\perp}\right| \ll \omega \quad \text { (relativistic) }
$$

and

$$
\Theta_{1} \simeq \Theta_{2} \ll 1 \quad \text { (small mixing) }
$$

In this limit the momenta for the two solutions read

$$
\begin{aligned}
& p_{1}=\omega-\Delta_{0}-\frac{g_{0}^{2} B_{T}^{2}}{4\left(\Delta_{0}+\Delta_{\perp}\right)} \\
& p_{2}=\omega+\Delta_{\perp}+\frac{g_{0}^{2} B_{T}^{2}}{4\left(\Delta_{0}+\Delta_{\perp}\right)},
\end{aligned}
$$

where we do not display higher-order terms that are not relevant in the approximation we work. Introducing these values in Eq. (15) shows that the solutions 1 and 2 are, respectively, $\phi$-like and photon-like. Let us also define

$$
\Theta \equiv \frac{1}{2} \frac{g_{0} B_{T}}{\Delta_{0}+\Delta_{\perp}} \simeq \Theta_{1} \simeq \Theta_{2}
$$

The solution to the linearized equations of motion Eqs. (8)-(9) would be given by a linear combination of the two found solutions

$$
\begin{aligned}
A_{\perp}(z) & =C_{1} \widetilde{A}_{\perp}^{(1)} e^{-i p_{1} z}+C_{2} \widetilde{A}_{\perp}^{(2)} e^{-i p_{2} z} \\
\phi(z) & =C_{1} \widetilde{\phi}^{(1)} e^{-i p_{1} z}+C_{2} \widetilde{\phi}^{(2)} e^{-i p_{2} z}
\end{aligned}
$$

with the integration constants $C_{1}$ and $C_{2}$ determined by the initial conditions.

Now we shall consider the conditions relevant for laboratory experiments where we start with some finite amplitude $A(0)$ and with $\phi(0)=0$ and we are interested in the fields after propagating some distance $z$. Solving for these initial conditions, we get

$$
\begin{aligned}
A_{\perp}(z) & =A_{\perp}(0)\left[\left(1-\Theta^{2}\right) e^{-i p_{2} z}+\Theta^{2} e^{-i p_{1} z}\right] \\
\phi(z) & =A_{\perp}(0) \Theta\left[e^{-i p_{2} z}-e^{-i p_{1} z}\right]
\end{aligned}
$$

where $p_{1}$ and $p_{2}$ are given by Eq. (20).

These expressions for the fields can be further simplified when taking the limit

$$
g_{0}^{2} B_{T}^{2} z \ll\left|\Delta_{0}+\Delta_{\perp}\right| .
$$

\footnotetext{
${ }^{4}$ When we consider the non relativistic limit $\omega \rightarrow m_{0}$ (not linearizing the e.o.m. (5) ) then $\tan \Theta_{1} \rightarrow$ 0 while $\tan \Theta_{2}$ remains finite and the difference between the scalar and pseudoscalar case is maximal. This limit has been carefully discussed for pseudoscalars in Ref. 47.
} 
We get, at the first non-trivial order in $\Theta$,

$$
\begin{aligned}
A_{\perp}(z) & =A_{\perp}(0) e^{-i \omega n_{\perp} z}\left[1-\Theta^{2}\left(2 \sin ^{2} \frac{b z}{2}+i(b z-\sin b z)\right)\right] \\
\phi(z) & =A_{\perp}(0) e^{-i \omega n_{\perp} z} \Theta\left[1-e^{i b z}\right]
\end{aligned}
$$

where

$$
b=\Delta_{0}+\Delta_{\perp} .
$$

From Eqs. (11)-(26) we see that the different photon components behave in a different way along their trajectory in the magnetic field. We can parameterize this difference with a factor that accounts for the change of the ratio of the amplitudes and a phase difference as follows:

$$
\frac{A_{\perp}(z)}{A_{\|}(z)}=\frac{A_{\perp}(0)}{A_{\|}(0)}(1-\eta(z)) e^{-i \varphi(z)},
$$

where $\eta(z)$ and $\varphi(z)$ are real and defined to be positive. We shall set $n_{\|}=n_{\perp}=1$ to focus on the effects of scalar-photon mixing; in this case $b=\Delta_{0}$.

The effects of the mixing are a decrease of the amplitude of the wave $A_{\perp}$ relative to $A_{\|}$given by

$$
1-\eta(z)=1-2 \Theta^{2} \sin ^{2} \frac{\Delta_{0} z}{2}
$$

and a phase delay given by

$$
\varphi(z)=\Theta^{2}\left(\Delta_{0} z-\sin \Delta_{0} z\right)
$$

To this extent, the vacuum filled with a magnetic field acts as a dichroic and birefringent medium. The consequences for a linearly polarized wave are: 1) a rotation of its polarization plane and 2) a small induced ellipticity (as long as the direction of polarization is not one of the preferred axes, $\perp$ or $\|)$.

We define the angle of polarization with respect to the external magnetic field as $\theta=\arctan \frac{\left|A_{\perp}\right|}{\left|A_{\mid}\right|}$. We can take it in the range $0 \leq \theta \leq \pi / 2$ since the sign of the magnetic field does not enter in any observable. Then a small change of $\left|A_{\perp}\right|$ and/or $\left|A_{\|}\right|$will produce a small rotation $\delta \theta$ given by

$$
\delta \theta(z)=\frac{\sin 2 \theta}{2}\left(\frac{\delta\left|A_{\perp}\right|}{\left|A_{\perp}\right|}-\frac{\delta\left|A_{\|}\right|}{\left|A_{\|}\right|}\right)=-\frac{\sin 2 \theta}{2} \eta(z),
$$

i.e. the polarization of the laser (its electric field) approaches the magnetic field plane. We can understand this as an effect of the depletion of photons perpendicularly polarized $A_{\perp}$ with respect to those polarized along the magnetic field direction $A_{\|}$. Pseudoscalar particles, such as axions, couple instead to $A_{\|}$and thus $\eta$ would be negative 
and their production would produce positive rotations of the laser polarization plane, i.e. the polarization of the laser would tend to be perpendicular to the magnetic field plane.

The ellipticity $\psi$ of a light wave is defined as

$$
\psi=\frac{A_{s}}{A_{l}}
$$

where $A_{s}\left(A_{l}\right)$ is the shorter (longer) axis of the ellipse that the vector of the electric field draws in the plane perpendicular to the propagation. As for the sign, we adopt the same conventions of Ref. [50], positive ellipticity meaning that the electric field follows the polarization ellipse in a clockwise sense as seen by an observer who sees the light propagating towards him. If the light is initially linearly polarized then a small phase delay $\varphi$ between $A_{\perp}$ and $A_{\|}$will lead to a shift of the ellipticity

$$
\delta \psi(z)=\delta\left(\frac{A_{s}}{A_{l}}\right)=-\frac{\sin 2 \theta}{2} \varphi(z) .
$$

The ellipticity is anti-clockwise because $A_{\perp}$ is delayed in time with respect to $A_{\|}$due to the fact that a small part of the $A_{\perp}$ wave travels now with a massive $\phi$-like dispersion relation. When this delay takes place $A_{\|}$reaches its maximum a bit before $A_{\perp}$ when this is still growing. Thus in the maximum of the amplitude the electric field moves away from the magnetic field vector. Notice that if the initial polarization is at an angle of $\pi / 4$ both the rotation $\delta \theta$ and the ellipticity $\delta \psi$ are maximized.

Since the transition is optimized if the scalar and photon waves are coherent, we think it is interesting to calculate the rotation and ellipticity in that limit, which corresponds to $\Delta_{0} z \ll 1$. Indeed $\Delta_{0}$ is the momentum difference in vacuum between a photon and a $\phi$ both with energy $\omega$, which generates, as the two quanta propagate along the $z$-direction, a relative phase of $\Delta_{0} z$. Requiring this phase to be negligible is precisely the coherence condition. In this limit the rotation and ellipticity reduce to:

$$
\begin{aligned}
& \delta \theta=-\frac{1}{16} g_{0}^{2} B_{T}^{2} z^{2} \sin 2 \theta \quad \text { (coherent) } \\
& \delta \psi=-\frac{1}{96} \frac{1}{\omega} g_{0}^{2} B_{T}^{2} m_{0}^{2} z^{3} \sin 2 \theta \quad \text { (coherent) . }
\end{aligned}
$$

As expected, both the rotation and ellipticity have opposite sign with respect to the ones generated by mixing with pseudoscalars. Except for this peculiarity, the phenomenology of this mixing is essentially equal to the axion case.

So far we have considered the case where an initial photon wave propagates along a magnetic field and is partially converted into a $\phi$ wave. If we consider the opposite case, i.e. an initially $\phi$ wave with no photon-component, we expect exactly the same 
solutions, Eqs. (23)-(24), but interchanging $A_{\perp} \leftrightarrow \phi$. This results can be used to compute the output of a "light-shining-through-walls" (LSW) experiment. In such an experiment a laser beam is shone through a magnetic field onto a thick wall where photons are stopped but $\phi$ 's potentially produced during the crossing of the magnetic field can traverse. By the inverse process, $\phi^{\prime} s$ can be reconverted into photons in another magnetic field behind the wall and detected in a low background environment. Plugging Eq. (27) and its equivalent in the inverse $\phi \rightarrow \gamma$ process we find a transmitted wave after the wall

$$
A_{\perp}\left(z_{c} ; z_{r}\right)=A_{\perp}(0) e^{-i \omega\left(z_{c}+z_{r}\right)} \Theta^{2}\left[1-e^{i b z_{c}}\right]\left[1-e^{i b z_{r}}\right]
$$

where $z_{c}, z_{r}$ are the lengths of the magnetic fields for the conversion and reconversion. This leads to a photon regeneration probability

$$
P\left(\gamma_{\perp} \rightarrow \phi \rightarrow \gamma_{\perp}\right)=\left|A_{\perp}\right|^{2}=16 \Theta^{4} \sin ^{2} \frac{b z_{c}}{2} \sin ^{2} \frac{b z_{r}}{2}
$$

i.e. the same expression as for pseudoscalars when the initial photons are polarized along the magnetic field direction. Under coherent conditions this is independent of the $\phi$ mass:

$$
P\left(\gamma_{\perp} \rightarrow \phi \rightarrow \gamma_{\perp}\right)=\frac{1}{16}\left(g_{0} B_{T} z_{c}\right)^{2}\left(g_{0} B_{T} z_{r}\right)^{2} \quad \text { (coherent) }
$$

\section{Mixing of Photons with Spin-Two Particles}

The Lagrangian describing a free massive spin-two particle $\chi$ was found by Fierz and Pauli in 1939 [51]:

$$
\begin{aligned}
\mathcal{L}_{F P}= & \frac{1}{4}\left(\partial_{\rho} \chi_{\mu \nu}\right)\left(\partial^{\rho} \chi^{\mu \nu}\right)-\frac{1}{2}\left(\partial_{\mu} \chi^{\mu \nu}\right)\left(\partial^{\rho} \chi_{\rho \nu}\right)+\frac{1}{2}\left(\partial_{\rho} \chi^{\rho \nu}\right)\left(\partial_{\nu} \chi_{\mu}^{\mu}\right) \\
& -\frac{1}{4}\left(\partial_{\nu} \chi_{\mu}^{\mu}\right)\left(\partial^{\nu} \chi_{\mu}^{\mu}\right)-\frac{m^{2}}{4} \chi_{\mu \nu} \chi^{\mu \nu}+\frac{m^{2}}{4}\left(\chi_{\mu}^{\mu}\right)^{2} .
\end{aligned}
$$

The equations of motion for $\chi_{\mu \nu}$ can be combined to give

$$
\partial_{\mu} \chi^{\mu \nu}=0 \quad \text { and } \quad \chi_{\mu}^{\mu}=0
$$

which are the usual constraints which apply to the rank-two tensor describing a spin-two particle, together with the requirement that $\chi_{\mu \nu}$ is symmetric. We observe that these constraints are dynamical conditions deriving from the Lagrangian itself. From the ten independent entries of a symmetric tensor, conditions (41) eliminate five, leaving the five degrees of freedom that represent the spin-two particle. 
In our study of the mixing we therefore decompose the field $\chi$ as

$$
\chi^{\mu \nu}(x)=\sum_{i} \chi_{i}(x) \epsilon_{i}^{\mu \nu}(p)
$$

where $\chi_{i}(x)$ are plane-waves and $\epsilon_{i}^{\mu \nu}$ are polarization tensors built to satisfy Eq. (41). Here $i$ runs therefore over the five spin-two polarizations: $+2, \times 2,+1, \times 1,0$. The explicit formulas for $\epsilon_{i}^{\mu \nu}$ are shown in Appendix A.

Next we consider an interaction Lagrangian of the type

$$
g \chi^{\mu \nu} O_{\mu \nu},
$$

where $O^{\mu \nu}$ is a bilinear in electromagnetic fields and $g$ a coupling constant.

Starting with the case of parity even spin-two particle, we have in principle two dimension-four operators candidates for $O^{\mu \nu}: F^{\mu \alpha} F_{\alpha}{ }^{\nu}$ and $\eta^{\mu \nu} F^{\alpha \beta} F_{\alpha \beta}$. Clearly, the second couples to the trace of $\chi_{\mu \nu}$, which is zero for our spin-two particle, and therefore cannot lead to any effect.

On the other hand, if we want our particle to be parity-odd, we find two analogous candidates. As we demonstrate in Appendix B, they are proportional to each other:

$$
F^{\mu \alpha} \widetilde{F}_{\alpha}^{\nu}=-\frac{1}{4} \eta^{\mu \nu} F^{\alpha \beta} \widetilde{F}_{\alpha \beta}
$$

and since one explicitly couples to the trace $\chi_{\mu}^{\mu}$ we conclude that mixing of photons with parity-odd spin-two particles should happen via higher-dimensional operators, which will necessarily include higher orders of the coupling constant $g$.

After this discussion, we are led to considering the mixing of a parity-even spin-two particle with the following lagrangian

$$
\mathcal{L}=\mathcal{L}_{E M}+\mathcal{L}_{F P}+\frac{g}{2 \sqrt{2}} \chi_{\mu \nu} F_{\alpha}^{\mu} F^{\alpha \nu}
$$

where we have defined the coupling constant with an appropriate numerical coefficient to make easier the comparison with the scalar case.

The equations of motion in the Lorentz gauge are, once projected onto the spin-two polarizations,

$$
\begin{aligned}
\left(\partial^{2}+m^{2}\right) \chi_{i} & =\sqrt{2} g \epsilon_{i}^{\mu \nu} F_{\mu \alpha}^{e x t}\left(\partial^{\alpha} A_{\nu}-\partial_{\nu} A^{\alpha}\right) \\
\partial^{2} A^{\nu} & =-\sqrt{2} g \sum_{i}\left(\epsilon_{i}^{\mu \alpha} F_{\alpha}^{e x t}{ }_{\alpha}^{\nu}-\epsilon_{i}^{\nu \alpha} F_{\alpha}^{e x t}{ }_{\alpha}^{\mu}\right) \partial_{\mu} \chi_{i} .
\end{aligned}
$$

We follow the same procedure as in the scalar case in Section 2 and shall work under the same conventions and assumptions. We linearize and redefine $A_{i} \rightarrow i A_{i}$ and we 
obtain the following equations in Fourier space:

$$
\begin{aligned}
& (\omega-p-\Delta) \tilde{\chi}_{\times 1}=0 \\
& (\omega-p-\Delta) \tilde{\chi}_{+1}=0 \\
& \left(\begin{array}{cc}
\omega-p-\Delta & a_{2} p \\
a_{2} p & \omega-p+\Delta_{\|}
\end{array}\right)\left(\begin{array}{c}
\widetilde{\chi}_{\times 2} \\
\widetilde{A}_{\|}
\end{array}\right)=\left(\begin{array}{l}
0 \\
0
\end{array}\right) \\
& \left(\begin{array}{ccc}
\omega-p-\Delta & 0 & a_{2} p \\
0 & \omega-p-\Delta & a_{0} p \\
a_{2} p & a_{0} p & \omega-p+\Delta_{\perp}
\end{array}\right)\left(\begin{array}{c}
\widetilde{\chi}_{+2} \\
\widetilde{\chi}_{0} \\
\widetilde{A}_{\perp}
\end{array}\right)=\left(\begin{array}{l}
0 \\
0 \\
0
\end{array}\right),
\end{aligned}
$$

where we have defined

$$
\Delta=\frac{m^{2}}{2 \omega}
$$

and

$$
a_{2}=\frac{g B_{T}}{2 \omega} \quad, \quad a_{0}=-\frac{g B_{T}}{\sqrt{3} \omega} .
$$

This system has seven states that could in principle mix, five for $\chi$ and two for $A$. However, we notice several interesting features. First of all, only the component $B_{T}$ and the states $\chi_{\times 2}, \chi_{+2}$ and $\chi_{0}$ appear in the mixing equations: from Eq. (48)-(49) we see indeed that $\chi_{\times 1}$ and $\chi_{+1}$ decouple. The same argument that we used in the scalar case can be used here to understand that $B_{L}$ cannot produce transitions to none of the states $\chi_{\times 2}, \chi_{+2}$ and $\chi_{0}$. In principle $B_{L}$ could produce transitions to the $\chi_{\times 1}$ and $\chi_{+1}$ (and not $B_{T}$, in this case). However, the states $\chi_{\times 1}$ and $\chi_{+1}$ have decoupled and do not appear in the mixing equations. This can be understood using angular momentum conservation, in a way reminiscent of the Landau-Yang theorem [23, 24]. Consider a massive spin-two particle at rest that decays into two photons and define the $z$-axis as the direction of propagation of the photons. It is clear that the spin-two particle cannot be in a spin state $s_{z}=+1$ nor $s_{z}=-1$ because the photons in the final state can only give $s_{z}= \pm 2$ or $s_{z}=0$. By boosting in the $z$ direction, the same result still holds, so that the particle is decoupled from the photons if it is in a $\chi_{\times 1}$ and $\chi_{+1}$ state.

Furthermore, we see is that the system has decoupled in two blocks, Eqs. (50)(51). This can be understood in terms of CP symmetry (see Appendix A). We follow Ref. [30] and define $\mathrm{P}$ as a reflection in the plane that contains $\vec{B}$ and the beam (plane $x-z)$. The magnetic field has $\mathrm{C}=-1$ and it is a pseudovector, so that $\mathrm{CP}=+1$. The photon field has also $\mathrm{C}=-1$ and the vector character implies $\mathrm{CP}=+1$ for $A_{\perp}$ and $\mathrm{C}=-1$ for $A_{\|}$. Finally, for the $\chi$ particle, we have that the polarizations $\times$ correspond to $\mathrm{CP}$ odd states, while + and 0 to even ones. All that implies that $A_{\perp}$ couples to + and 0 while $A_{\|}$to $\times$. We can now fully understand the convenience of choosing the $\times$ and + polarizations as we have done.

An interesting result we have obtained is that the $A_{\perp}-\chi_{0}$ mixing, $a_{0}$, is of the same order of magnitude as $a_{2}$ and, in particular, does not vanish when $m \rightarrow 0$. In Ref. [31, 
the helicity-0 contribution was neglected, based on the analogy with the massless case (standard general relativity) considered in Ref. [27].

The structure of the mixing matrix in Eq. (51) allows us to perform a simple rotation in the polarization states space of $\chi$ in such a way that only a linear combination of the spin-two states couples to the photon. The combination that couples to $A_{\perp}$ is

$$
\chi_{+}=\frac{a_{2}}{a_{+}} \chi_{+2}+\frac{a_{0}}{a_{+}} \chi_{0}
$$

with

$$
a_{+}=\sqrt{a_{2}^{2}+a_{0}^{2}}
$$

In terms of this linear combination, the problem reduces to a two-particle mixing. Instead of Eq. (51), we have

$$
\left(\begin{array}{cc}
\omega-p-\Delta & a_{+} p \\
a_{+} p & \omega-p+\Delta_{\perp}
\end{array}\right)\left(\begin{array}{c}
\widetilde{\chi}_{+} \\
\widetilde{A}_{\perp}
\end{array}\right)=\left(\begin{array}{l}
0 \\
0
\end{array}\right) .
$$

The orthogonal combination $\chi_{+}^{\prime}=\left(a_{0} \chi_{+2}-a_{2} \chi_{0}\right) / a_{+}$decouples, so we end up with a simple two-by-two mixing problem for every photon polarization. The solutions can be read directly from the scalar case with appropriate momenta and mixing angles. As we said, we work in the same approximations than in the scalar case, that here read $\Delta,\left|\Delta_{\|}\right|,\left|\Delta_{\perp}\right| \ll \omega$ (already needed when linearizing the equations of motion) and $g^{2} B_{T}^{2} /\left(\Delta+\Delta_{i}\right)^{2} \ll 1$, for both $i=\|, \perp$.

Using Eq. (20) we find the momenta in the spin-two case:

$$
\begin{aligned}
& p_{\times}^{(1)}=\omega-\Delta-\frac{a_{\times}^{2}}{b_{\times}} \omega^{2} \quad, \quad p_{\times}^{(2)}=\omega+\Delta_{\|}+\frac{a_{\times}^{2}}{b_{\times}} \omega^{2} \\
& p_{+}^{(1)}=\omega-\Delta-\frac{a_{+}^{2}}{b_{+}} \omega^{2} \quad, \quad p_{+}^{(2)}=\omega+\Delta_{\perp}+\frac{a_{+}^{2}}{b_{+}} \omega^{2}
\end{aligned}
$$

where, to unify notation, we have defined $a_{\times}=a_{2}$ and also

$$
b_{\times}=\Delta+\Delta_{\|} \quad, \quad b_{+}=\Delta+\Delta_{\perp} .
$$

The mixing angles are

$$
\Theta_{\times}=\frac{a_{\times}}{b_{\times}} \omega \quad, \quad \Theta_{+}=\frac{a_{+}}{b_{+}} \omega .
$$

The solutions for $\left(A_{\|}, \chi_{\times}\right)$and $\left(A_{\perp}, \chi_{+}\right)$are completely analogous to Eqs. (23)-(24). 
In the limit $g^{2} B_{T}^{2} z \ll\left|\Delta+\Delta_{i}\right| \ll 1$, for both $i=\|, \perp$ we have

$$
\begin{aligned}
& A_{\|}(z)=A_{\|}(0) e^{-i \omega n_{\|} z}\left[1-\Theta_{\times}^{2}\left(2 \sin ^{2} \frac{b_{\times} z}{2}+i\left(b_{\times} z-\sin b_{\times} z\right)\right)\right] \\
& A_{\perp}(z)=A_{\perp}(0) e^{-i \omega n_{\perp} z}\left[1-\Theta_{+}^{2}\left(2 \sin ^{2} \frac{b_{+} z}{2}+i\left(b_{+} z-\sin b_{+} z\right)\right)\right] \\
& \chi_{\times}(z)=A_{\|}(0) e^{-i \omega n_{\|} z} \Theta_{\times}\left[1-e^{i b_{\times} z}\right] \\
& \chi_{+}(z)=A_{\perp}(0) e^{-i \omega n_{\perp} z} \Theta_{+}\left[1-e^{i b_{+} z}\right] \\
& \chi_{+}^{\prime}(z)=0 .
\end{aligned}
$$

As in the scalar case, we set $n_{\|}=n_{\perp}=1$ to focus on the effects of photon- $\chi$ mixing so that $b_{\times}=b_{+}=\Delta$. Then, the relative amplitude change and phase delay of $A_{\perp}$ and $A_{\|}$are now given by

$$
\begin{gathered}
1-\eta(z)=1-2 \frac{a_{0}^{2} \omega^{2}}{\Delta^{2}} \sin ^{2} \frac{\Delta z}{2} \\
\varphi(z)=\frac{a_{0}^{2} \omega^{2}}{\Delta^{2}}(\Delta z-\sin \Delta z) .
\end{gathered}
$$

We see that the effects of production of the $2+$ and $2 \times$ polarizations cancel out 5 , leaving only the effect caused by $A_{\perp} \rightarrow \chi_{0}$ transitions. We could have expected this since the $A_{\perp}-\chi_{2+}$ and $A_{\|}-\chi_{2 \times}$ mixing in vacuum is driven by the same $a_{2}$ (see Eqs. (50)-(51) ) but $A_{\perp}$ can also convert into $\chi_{0}$ and thus depletes and delays its phase faster than $A_{\|}$.

Note that in the massless case $\chi_{2+}$ and $\chi_{2 \times}$ are the only physical components and therefore the cancellation of their effects does not lead to any net effect neither in the rotation nor in the ellipticity. However, in the $m \neq 0$ case and even with a vanishingly small value for $m$, the 0-polarization does not decouple and we expect indeed both effects. The corresponding formulas in the coherent case are given by

$$
\begin{array}{rlr}
\delta \theta & =-\frac{1}{12} g^{2} B_{T}^{2} z^{2} \sin 2 \theta & \text { (coherent) } \\
\delta \psi & =-\frac{1}{72} \frac{1}{\omega} g^{2} B_{T}^{2} m^{2} z^{3} \sin 2 \theta & \text { (coherent) }
\end{array}
$$

i.e. the same we got for a spin-zero particle, except for a factor $4 / 3$ that can be traced back to Eq. (53). This factor can be reabsorbed in a redefinition of the coupling constant $g$, so that, from a positive measurement of $\delta \theta$ and $\delta \psi$ we would not be able to distinguish between the scalar and the massive spin-two case.

\footnotetext{
${ }^{5}$ This statement turns out to be slightly modified at the one loop level [28, 29].
} 
On the other hand the LSW probability differs from the scalar case, since here it is non-zero for both photon polarizations. Using the analogous of Eq. (37), in the coherent limit, it is given by

$$
\begin{aligned}
& P\left(\gamma_{\perp} \rightarrow \chi \rightarrow \gamma_{\perp}\right)=\left(a_{0}^{2}+a_{2}^{2}\right)^{2} \omega^{4} z_{c}^{2} z_{r}^{2}=\frac{49}{9} \frac{1}{16}\left(g B_{T}\right)^{4} z_{c}^{2} z_{r}^{2} \\
& P\left(\gamma_{\|} \rightarrow \chi \rightarrow \gamma_{\|}\right)=\quad a_{2}^{4} \omega^{4} z_{c}^{2} z_{r}^{2} \quad=\frac{1}{16}\left(g B_{T}\right)^{4} z_{c}^{2} z_{r}^{2} .
\end{aligned}
$$

Note that perpendicular photons have a factor $(1+4 / 3)^{2}=49 / 9 \sim 5$ more chances to traverse the wall. Physically this comes from the fact that the passage through the wall as a $\chi_{0}$ and as $\chi_{2+}$ adds up coherently at the amplitude level.

\section{Discussion and Conclusions}

To summarize, in this paper we have considered the mixing of photons with massive scalars $\phi$ and with massive spin-two particles $\chi$ that arises in the presence of a magnetic field. Starting with the Lagrangian that contains the coupling to two photons, we have calculated the mixing matrices and the effects on light propagating in a magnetic field.

The mixing equations of the scalar-photon system present some differences with the well-studied axion-photon system. Technically, this is due to the fact than in the scalar case the interaction term, i.e. the r.h.s. of Eqs. (5) - (6) ), contains spatial derivatives of the fields, while in the pseudoscalar case the derivatives are with respect to time. This means that we can pass from the propagation eigenstates in vacuum ( $\phi$ and $A_{i}$ ) to the ones in the magnetic field by performing a transformation which is not a simple rotation. While this introduces a formal difference between the two cases, in practice this difference disappears when we take the relativistic limit. These same issues appear in the spin-two case we have developed in Section 3. Actually, an analogous situation occurs in the axion-photon mixing in an electric field. In this case the interaction lagrangian is $\propto a B \cdot E$. While in a constant external magnetic field the axion-photon mixing results to be $\propto \partial_{0} A \cdot B_{\text {ext }}=i \omega A_{\|} B_{\text {ext }}$, in an external electric field is $\propto(\nabla \times A) \cdot E_{\text {ext }}=-i p A_{\perp} E_{\text {ext }}$, in analogy with the case discussed here.

When constructing the interaction Lagrangian, we have seen that the interaction of a $2^{+}$particle is a dimension-five operator, as expected. However, the interaction operators in the $2^{-}$case are at least dimension-seven, so that they will be much more suppressed. For this reason we have only calculated the parity-even case.

Referring now to the mixing of a $2^{+}$particle with photons, we have seen that the helicity-one states $\chi_{1}$ decouple. This can be understood with arguments of rotational symmetry, as explained in Section 3. Apart from the decoupling just mentioned, there is further decoupling since $A_{\|}$couples only to the $\times 2$ mode while $A_{\perp}$ couples only 
to the +2 and 0 modes. As also explained in Section 3 , it is a consequence of CP symmetry. We have seen that the $A_{\perp}-\chi_{2}$ and the $A_{\|}-\chi_{2}$ mixing have identical value, but $\chi_{0}$ makes a difference: it mixes with $A_{\perp}$ only. As a consequence, the contributions to the rotation and ellipticity observables of $\gamma-\chi$ mixing contain only the $A_{\perp}-\chi_{0}$ mixing. Moreover, compared to the $A-\chi_{2}$ mixing amplitude, the $A-\chi_{0}$ mixing is not suppressed, indeed it is larger of a factor $\sqrt{4 / 3}$.

In the eventual discovery of effects of rotation and ellipticity in light propagating in a magnetic field, and in the eventual case that the signs would correspond to a parity-even particle, the tensor case should be considered as a possible explanation, together of course with the scalar case. In such a case, a "light-shining-through-walls" experiment would be useful to discriminate between these two candidates since photons with polarization parallel to the magnetic field would lead to a signal only in the spintwo case. This is because, in this case, both polarizations would lead to positive signals. Moreover, we have found that the probability for photons polarized perpendicularly to the magnetic field is a factor $(1+4 / 3)^{2}$ larger than for photons with parallel polarization, due to the additional $\chi_{0}$ intermediate state.

While in the massless spin-two case there are no observable effects of rotation and ellipticity, we have found that there are effects when taking the $m \rightarrow 0$ limit of the massive theory because the $A-\chi_{0}$ mixing does not vanish. Our particle could be a massive graviton but it could be as well a tensorial particle with no relation

whatsoever with gravitation. This result might be of theoretical interest in the light of the controversy outlined in Section 1 .

\section{Acknowledgements}

We would like to thank D. Blas, E. Álvarez and P. Silva for discussions. We acknowledge support by the CICYT Research Projects FPA2003-04597 and FPA2005-05904, the Departament d'Universitats, Recerca $i$ Societat de la Informació (DURSI), Project 2005SGR00916 and the European Commision under the RTN program MRTN-CT2004-503363. C.B. is grateful to IFAE (Universitat Autònoma de Barcelona) for hospitality while part of the work was performed. 


\section{Appendixes}

\section{A Polarization of a spin-two particle}

It has been known since a long time [52] how to construct the polarization basis of particles with arbitrary spin starting from spin one-half and one. In particular spintwo can be built from spin-one. For a vector particle propagating along $z$ with fourmomentum $P^{\mu}=(\omega ; 0,0, p)$, the helicities $+1,-1$ and 0 can be chosen to be

$$
\begin{aligned}
\epsilon^{\mu}(h= \pm 1) & =(0, \mp 1,-i, 0) \\
\epsilon^{\mu}(h=0) & =(p / m, 0,0, \omega / m) .
\end{aligned}
$$

where here $m$ has to be implicitly understood as $m=\sqrt{P_{\mu} P^{\mu}}=\sqrt{\omega^{2}-p^{2}}$.

By using the appropriate Clebsch-Gordan coefficients, one can obtain the helicities $h=0, \pm 1, \pm 2$. For example, $h=+2$ is given by

$$
\epsilon^{\mu \nu}(h=+2)=\epsilon^{\mu}(h=+1) \epsilon^{\nu}(h=+1)=\frac{1}{2}\left(\begin{array}{cccc}
0 & 0 & 0 & 0 \\
0 & 1 & i & 0 \\
0 & i & -1 & 0 \\
0 & 0 & 0 & 0
\end{array}\right)
$$

and so on. For our calculations it is more convenient to work with CP-eigenstates. Since CP transforms $+h$ into $-h$, we will use the following combinations

$$
\begin{aligned}
\epsilon_{+2}^{\mu \nu} \quad & =\frac{-1}{\sqrt{2}}\left(\epsilon^{\mu \nu}(h=+2)+\epsilon^{\mu \nu}(h=-2)\right)=\frac{1}{\sqrt{2}}\left(\begin{array}{cccc}
0 & 0 & 0 & 0 \\
0 & -1 & 0 & 0 \\
0 & 0 & 1 & 0 \\
0 & 0 & 0 & 0
\end{array}\right) \\
\epsilon_{\times 2}^{\mu \nu} \quad & =\frac{1}{\sqrt{2} i}\left(\epsilon^{\mu \nu}(h=+2)-\epsilon^{\mu \nu}(h=-2)\right)=\frac{1}{\sqrt{2}}\left(\begin{array}{cccc}
0 & 0 & 0 & 0 \\
0 & 0 & 1 & 0 \\
0 & 1 & 0 & 0 \\
0 & 0 & 0 & 0
\end{array}\right) \\
\epsilon_{+1}^{\mu \nu} \quad= & \frac{1}{\sqrt{2} i}\left(\epsilon^{\mu \nu}(h=+1)+\epsilon^{\mu \nu}(h=-1)\right)=-\frac{1}{m \sqrt{2}}\left(\begin{array}{cccc}
0 & 0 & p & 0 \\
0 & 0 & 0 & 0 \\
p & 0 & 0 & \omega \\
0 & 0 & \omega & 0
\end{array}\right) \\
\epsilon_{\times 1}^{\mu \nu} \quad= & \frac{1}{\sqrt{2}}\left(\epsilon^{\mu \nu}(h=+1)-\epsilon^{\mu \nu}(h=-1)\right)=\frac{1}{m \sqrt{2}}\left(\begin{array}{cccc}
0 & p & 0 & 0 \\
p & 0 & 0 & \omega \\
0 & 0 & 0 & 0 \\
0 & \omega & 0 & 0
\end{array}\right)
\end{aligned}
$$




$$
\epsilon_{0}^{\mu \nu}=\epsilon^{\mu \nu}(h=0)=\frac{1}{m^{2}} \sqrt{\frac{2}{3}}\left(\begin{array}{cccc}
2 p^{2} & 0 & 0 & 2 p \omega \\
0 & -m^{2} & 0 & 0 \\
0 & 0 & -m^{2} & 0 \\
2 p \omega & 0 & 0 & 2 \omega^{2}
\end{array}\right) .
$$

Note that, as expected, for all $i=0,+1, \times 1,+2, \times 2$ the requirements of Eq. (41) are satisfied,

$$
P_{\mu} \epsilon_{i}^{\mu \nu}=0 \quad \epsilon_{i}{ }^{\mu}{ }_{\mu}=0 \quad \epsilon_{i}^{\mu \nu}=\epsilon_{i}^{\nu \mu}
$$

and also

$$
\epsilon_{\mu \nu i} \epsilon_{j}^{\mu \nu}=\delta_{i j} \quad \forall i, j
$$

\section{B A useful identity}

Given $A_{\mu \nu}$ and $B_{\mu \nu}$ antisymmetric we first evaluate

$$
\widetilde{A}_{\mu \nu} \widetilde{B}^{\mu \rho}=\frac{1}{4} \epsilon_{\mu \nu \alpha \beta} \epsilon^{\mu \rho \sigma \tau} A^{\alpha \beta} B_{\sigma \tau}
$$

Then we use

$$
\epsilon_{\mu \nu \alpha \beta} \epsilon^{\mu \rho \sigma \tau}=\left|\begin{array}{ccc}
\delta_{\nu}^{\rho} & \delta_{\alpha}^{\rho} & \delta_{\beta}^{\rho} \\
\delta_{\nu}^{\sigma} & \delta_{\alpha}^{\sigma} & \delta_{\beta}^{\sigma} \\
\delta_{\nu}^{\tau} & \delta_{\alpha}^{\tau} & \delta_{\beta}^{\tau}
\end{array}\right|
$$

to obtain

$$
\widetilde{A}_{\mu \nu} \widetilde{B}^{\mu \rho}=\frac{1}{2} \delta_{\nu}^{\rho} A_{\alpha \beta} B^{\alpha \beta}-A^{\alpha \rho} B_{\alpha \nu}
$$

We apply now the identity to $\widetilde{A}=F$ and $\widetilde{B}=\widetilde{F}$ :

$$
F_{\mu \nu} \widetilde{F}^{\mu \rho}=\frac{1}{2} \delta_{\nu}^{\rho} \widetilde{F}_{\alpha \beta} F^{\alpha \beta}-\widetilde{F}^{\alpha \rho} F_{\alpha \nu} ;
$$

since the last term is identical to the l.h.s., we finally obtain:

$$
F_{\mu \nu} \widetilde{F}^{\mu \rho}=\frac{1}{4} \delta_{\nu}^{\rho} \widetilde{F}_{\alpha \beta} F^{\alpha \beta}
$$

\section{References}

[1] H. Primakoff, Phys. Rev. 81 (1951) 899.

[2] P. Sikivie, Phys. Rev. Lett. 51 (1983) 1415. [Erratum-ibid. 52, 695 (1984)]. 
[3] S. Asztalos et al., Phys. Rev. D64 (2001) 092003.

[4] D. M. Lazarus et al., Phys. Rev. Lett. 69 (1992) 2333-2336.

[5] S. Moriyama et al., Phys. Lett. B434 (1998) 147, hep-ex/9805026.

[6] Y. Inoue et al., Phys. Lett. B536 (2002) 18-23, astro-ph/0204388.

[7] Y. Inoue et al., Phys. Lett. B668 (2008) 93-97, arXiv:0806.2230 [astro-ph].

[8] CAST Collaboration, K. Zioutas et al., Phys. Rev. Lett. 94 (2005) 121301, hep-ex/0411033.

[9] CAST Collaboration, S. Andriamonje et al., JCAP 0704 (2007) 010, hep-ex/0702006.

[10] BFRT Collaboration, G. Ruoso et al., Z. Phys. C56 (1992) 505-508.

[11] BRFT Collaboration, R. Cameron et al., Phys. Rev. D47 (1993) 3707-3725.

[12] PVLAS Collaboration, E. Zavattini et al., Phys. Rev. Lett. 96 (2006) 110406, hep-ex/0507107.

[13] PVLAS Collaboration, E. Zavattini et al., Phys. Rev. D77 (2008) 032006, arXiv:0706.3419 [hep-ex].

[14] GammeV (T-969) Collaboration, A. S. Chou et al., Phys. Rev. Lett. 100 (2008) 080402, arXiv:0710.3783 [hep-ex].

[15] BMV Collaboration, M. Fouche et al., Phys. Rev. D78 (2008) 032013 , arXiv:0808.2800 [hep-ex].

[16] Q\&A Collaboration, S.-J. Chen, H.-H. Mei, and W.-T. Ni, Mod. Phys. Lett. A22 (2007) 2815-2831, arXiv: hep-ex/0611050.

[17] ALPS Collaboration, K. Ehret et al., hep-ex/0702023.

[18] LIPSS Collaboration, A. Afanasev et al., Phys. Rev. Lett. 101 (2008) 120401, arXiv:0806.2631 [hep-ex].

[19] OSQAR Collaboration, P. Pugnat et al., arXiv:0712.3362 [hep-ex].

[20] G. Cantatore, R. Cimino, M. Karuza, V. Lozza, and G. Raiteri, arXiv:0809.4208 [hep-ex].

[21] Particle Data Group Collaboration, S. Eidelman et al., Phys. Lett. B592 (2004) 1. 
[22] E. Masso and R. Toldra, Phys. Rev. D52 (1995) 1755-1763, hep-ph/9503293.

[23] L. D. Landau, Dokl. Akad. Nauk SSSR 60 (1948) 207.

[24] C.-N. Yang, Phys. Rev. 77 (1950) 242-245.

[25] M. E. Gertsenshtein, Zh. Eksp. Teor. Fiz. 41 (1961) 109. [Sov. Phys. JETP 14, 81 (1962)].

[26] P. C. Aichelburg and R. U. Sexl, Gen. Rel. Grav. 2 (1971) 303-312.

[27] J. C. R. Magueijo, Phys. Rev. D49 (1994) 671-680.

[28] F. Bastianelli and C. Schubert, JHEP 02 (2005) 069, arXiv:gr-qc/0412095.

[29] F. Bastianelli, U. Nucamendi, C. Schubert, and V. M. Villanueva, JHEP 11 (2007) 099, arXiv:0710.5572 [gr-qc].

[30] G. Raffelt and L. Stodolsky, Phys. Rev. D37 (1988) 1237.

[31] C. Deffayet and J.-P. Uzan, Phys. Rev. D62 (2000) 063507, arXiv:hep-ph/0002129.

[32] G. G. Raffelt, Stars as laboratories for fundamental physics: The astrophysics of neutrinos, axions, and other weakly interacting particles. The University of Chicago Press, 1996. 664 p.

[33] G. G. Raffelt, Ann. Rev. Nucl. Part. Sci. 49 (1999) 163-216, hep-ph/9903472.

[34] H. Schlattl, A. Weiss, and G. Raffelt, Astropart. Phys. 10 (1999) 353-359, arXiv:hep-ph/9807476.

[35] E. G. Adelberger et al., Phys. Rev. Lett. 98 (2007) 131104. arXiv:hep-ph/0611223.

[36] S. Schlamminger, K. Y. Choi, T. A. Wagner, J. H. Gundlach, and E. G. Adelberger, Phys. Rev. Lett. 100 (2008) 041101, arXiv:0712.0607 [gr-qc]

[37] A. Dupays, E. Masso, J. Redondo, and C. Rizzo, Phys. Rev. Lett. 98 (2007) 131802, hep-ph/0610286.

[38] E. Masso and J. Redondo, JCAP 0509 (2005) 015, hep-ph/0504202.

[39] P. Brax, C. van de Bruck, and A.-C. Davis, Phys. Rev. Lett. 99 (2007) 121103, hep-ph/0703243.

[40] E. Masso and J. Redondo, Phys. Rev. Lett. 97 (2006) 151802, hep-ph/0606163. 
[41] R. N. Mohapatra and S. Nasri, Phys. Rev. Lett. 98 (2007) 050402, arXiv:hep-ph/0610068.

[42] A. S. Goldhaber and M. M. Nieto, arXiv:0809.1003 [hep-ph].

[43] H. van Dam and M. J. G. Veltman, Nucl. Phys. B22 (1970) 397-411.

[44] V. I. Zakharov, JETP Lett. 12 (1970) 312.

[45] A. I. Vainshtein, Phys. Lett. B39 (1972) 393-394.

[46] F. Hoogeveen and T. Ziegenhagen, Nucl. Phys. B358 (1991) 3-26.

[47] S. L. Adler, J. Gamboa, F. Mendez, and J. Lopez-Sarrion, Annals Phys. 323 (2008) 2851-2872, arXiv:0801.4739 [hep-ph].

[48] L. Maiani, R. Petronzio, and E. Zavattini, Phys. Lett. B175 (1986) 359.

[49] Y. Liao, Phys. Lett. B650 (2007) 257-261, arXiv:0704.1961 [hep-ph].

[50] M. Born and E. Wolf, Principles of Optics. Pergamon Press, 1980. 691-692.

[51] M. Fierz and W. Pauli, Proc. Roy. Soc. Lond. A173 (1939) 211-232.

[52] P. R. Auvil and J. J. Brehm, Phys. Rev. 145 (1966) 1152. 